\title{
Assessment of two different HER2 scoring systems and clinical relevance for colorectal cancer
}

\author{
Furong Liu ${ }^{1,2} \cdot$ Chao Ren ${ }^{1,2} \cdot$ Ying Jin ${ }^{1,2}$. Shaoyan $\mathrm{Xi}^{1,3} \cdot$ Caiyun $\mathrm{He}^{1,4} \cdot$ Fang Wang $^{1,4} \cdot$ Zixian Wang ${ }^{1,2} \cdot$ Rui-hua Xu $^{1,2}$. \\ Feng Wang ${ }^{1,2}$ (D)
}

Received: 1 August 2019 / Revised: 28 August 2019 / Accepted: 10 September 2019 / Published online: 13 November 2019

(C) The Author(s) 2019

\begin{abstract}
Although the positivity of human epidermal growth factor receptor 2 (HER2) is low in colorectal cancer (CRC), anti-HER2 is becoming a new target therapy in metastatic colorectal cancer (mCRC). However, assessment of the HER2 scoring system was still not established in CRC. The purpose of our study was to evaluate HER2 status and its correlation with clinicopathological characteristics and survival according to the HER2 diagnostic criteria for gastroesophageal adenocarcinoma (GEA criteria) and the HERACLES diagnostic criteria (HERACLES criteria) in a large cohort of Chinese CRC patients. The HER2 positivity was $2.9 \%$ (43/1490) and 2.6\% (39/1490) in CRCs based on the GEA criteria and the HERACLES criteria, and 3.7\% (9/243) in mCRC according to both criteria. HER2 status was associated with primary tumor location $(P=0.037)$, regional lymph node metastasis $(P=0.035)$, and TNM stage $(P=0.022)$ in CRCs based on the HERACLES criteria. No such association was found based on the GEA criteria. Furthermore, HER2 positive only presented in patients with RAS gene wild type $(P=0.001)$. Significant difference was only observed between the HER2-positive and HER2-negative groups in terms of disease-free survival for stage II-III CRCs $(P=0.048)$ according to the HERACLES criteria, but not based on the GEA criteria. Our findings suggest that the frequency of HER2 overexpression or amplification was low in Chinese CRC patients, and provide a rationale for further evaluation of HER2 in CRC based on the HERACLES criteria and the HER2 diagnostic criteria for gastroesophageal adenocarcinoma.
\end{abstract}

Keywords HER $2 \cdot$ Colorectal cancer $\cdot$ Methodology $\cdot$ Prognosis

Furong Liu and Chao Ren contributed equally to this work.

Electronic supplementary material The online version of this article (https://doi.org/10.1007/s00428-019-02668-9) contains supplementary material, which is available to authorized users.

Feng Wang

wangfeng@ sysucc.org.cn

1 Sun Yat-sen University Cancer Center, State Key Laboratory of Oncology in South China, Collaborative Innovation Center for Cancer Medicine, 651 Dongfeng Road East, Guangzhou 510060, People's Republic of China

2 Department of Medical Oncology, Sun Yat-sen University Cancer Center, 651 Dongfeng Road East, Guangzhou 510060, People's Republic of China

3 Department of Pathology, Sun Yat-sen University Cancer Center, 651 Dongfeng Road East, Guangzhou 510060, People's Republic of China

4 Department of Molecular Diagnostics, Sun Yat-sen University Cancer Center, 651 Dongfeng Road East, Guangzhou 510060, People's Republic of China

\section{Introduction}

Colorectal cancer (CRC) is the third most common malignant neoplasm and the second leading cause of cancer-related deaths worldwide [1]. It is estimated that 370,000 newly diagnosed cases of CRC and 180,000 deaths from CRC occurred in China in 2014 [2]. Approximately 20\% of the patients with newly diagnosed CRC already have distant metastases at the time of diagnosis and for those without metastasis [3], they can be treated with curative approaches but still retain a high risk of recurrence (up to 50\%) [4]. As such, advanced stage diseases and recurrences after curative treatment are a significant cause of death. The standard treatment for such patients is chemotherapy, and recently, the addition of targeted therapies has shown improved therapeutic outcomes. Cetuximab and panitumumab, which are directed against the epidermal growth factor receptor (EGFR), are the most common monoclonal antibody used in metastatic colorectal cancer $(\mathrm{mCRC})$ to prolong survival $[4,5]$. However, approximately 
$50 \%$ of CRC patients cannot benefit from the anti-EGFR treatments since their tumors harbor the RAS or BRAF mutations $[6,7]$. The biology of CRC is still far from being fully understood, and researchers are still striving to identify new biomarkers for potential therapeutic targets and novel approaches for predicting therapeutic responses and improving survival outcomes.

The human epidermal growth factor receptor 2 (HER2, also known as HER2/neu, C-erbB2, and p185) is a member of the EGFR family of receptor tyrosine kinases [8]. Over the past two decades, the status of HER2 has shown to have an important role in the development and progression of approximately $30 \%$ of breast cancer cases and $10 \%$ of gastric cancer cases [9-11]. Trastuzumab is the monoclonal antibody that acts by blocking the HER2 receptor, and its use has become a standard treatment for patients with breast and gastric cancer presenting with HER2 gene amplification or membranous HER2 protein overexpression [12, 13]. After the breakthroughs in breast and gastric cancers, the efficacy of antiHER2 therapy was evaluated in CRC. The HERACLES trial was a multicenter open-label phase II trial that enrolled patients with wild-type KRAS exon 2, HER2 overexpressing metastatic colorectal cancer refractive to chemotherapy, and anti-EGFR therapy resistance. The objective response rate (ORR) was $30 \%$ for patients received a combination of trastuzumab and lapatinib [14]. Therefore, they brought up criteria for HER2 assessment in CRC (HERACLES criteria). However, until present, other studies also used the HER2 diagnostic criteria for gastroesophageal adenocarcinoma (GEA criteria) for HER2 assessment in CRC [15-17]. Moreover, the relationship between the expression of HER2 and clinicopathological prognostic factors remains controversial [15, 17-20]. These debatable results indicate that the role of HER2 in CRC requires further exploration.

Thence, the purpose of this study was to compare the two different HER2 scoring systems of CRC in large Chinese patients and analyze the influence of HER2 status on clinicopathological factors and survival of CRC.

\section{Material and methods}

\section{Study design}

One thousand five hundred sixty CRC patients with formalin-fixed paraffin-embedded archival samples at Sun Yat-sen University Cancer Center from January 2016 to December 2017 were identified. One thousand five hundred fifteen patients enrolled by fulfilling the following inclusion criteria: (a) patients diagnosed as histologically prove CRC; (b) patients with complete clinical, pathological, and prognostic information. Twenty-five patients were excluded based on the following exclusion criteria: (a) lack of tumor tissue for further test (five cases); (b) presence of simultaneous carcinoma (11 patients); (c) refusal anti-tumor treatment (nine patients). Finally, 1490 eligible patients were enrolled in our analysis (Supplementary Fig. 1). The institutional review board approved the study, and informed consent was waived. All clinicopathologic data were obtained from the patients' records.

\section{HER2 expression and amplification tests}

HER2 expression analysis was performed by immunohistochemistry (IHC) using primary monoclonal antibody against HER2/neu (clone CB-11, dilution 1:65, Cell Marque, Rocklin, CA, USA), following the manufacturer's protocol. The procedure was carried out in our Department of Pathology. All tumor tissue specimens were processed by the hematoxylin and eosin (H\&E) method, and the sections comprising at least $70 \%$ tumor cells were cut into $4-\mu \mathrm{m}$ sections for IHC staining. The HER2 immunoreactivity was presented with percentage and intensity. The intensity of reactivity was grouped as IHC score 0 , no reactivity or membrane staining; IHC score $1+$, faint/barely perceptible partial membrane staining; IHC score $2+$, weak to moderate complete or basolateral membrane staining; and IHC score $3+$, moderate to strong complete membrane staining [21]. IHC analysis was performed by one trained pathologist. Ambiguous cases were reanalyzed by a second pathologist.

HER2 amplification analysis was performed by fluorescent in situ hybridization (FISH) using the FDA-approved Vysis PathVysion HER-2/neu DNA Probe Kit (Dako Cytomation, Denmark). The scoring was carried out in no less than 20 nonoverlapping nuclei core in tumor regions. A ratio of HER2 signal to CEP17 signal of $\geq 2$ was considered amplification of HER2 [21].

\section{Assessment of HER2 status}

\section{The HER2 diagnostic criteria for gastroesophageal adenocarcinoma (GEA criteria)}

HER2-positive cases were those who had an IHC score of $3+$ in more than $10 \%$ of the tumor cells or for those who had an IHC score $2+$ in more than $10 \%$ of the tumor cells and demonstrated positive amplification of HER2 gene by FISH [21].

\section{The HERACLES diagnostic criteria (HERACLES criteria)}

The HERACLES criteria of HER2 positive were tumors with a $3+$ HER 2 score in more than $50 \%$ of the tumor cells by IHC or with a $3+$ HER 2 score in $10 \sim 50 \%$ of the tumor cells by IHC and FISH positive, or with a $2+$ HER 2 score two in more than $50 \%$ of the tumor cells by IHC and FISH positive. FISH 
positivity was defined as a HER2:CEP17 ratio higher than two in more than $50 \%$ of the tumor cells [14].

\section{Follow-up}

All patients underwent continuous follow-up in accordance with that specified in the National Comprehensive Cancer Network (NCCN) guidelines. The disease-free survival (DFS) for patients with stage II-III was defined as the time from the first day of surgery until the first documented recurrence date, or death from any cause or the time censored. The progression-free survival (PFS) for mCRC patients was defined as the time from the first day of treatment until the first documented progressive disease (PD) according to Response Criteria Evaluation in Solid Tumors (RECIST) version 1.1 or death from any cause, whichever occurred first for mCRC [22]. The censor date was December 31, 2018.

\section{Statistical analysis}

Comparisons were made using $t$ test, the chi-square test, and Fisher exact test, as appropriate. The survival was analyzed using the Kaplan-Meier method and the log-rank test was used to determine the significance of the difference between the survival curves. Univariate analysis and multivariate analysis were performed by the Cox proportional hazards regression model to determine the significant prognostic factors on survival. All tests were two-tailed, and statistical significance was set at $P<0.05$. The statistical analysis of the data was performed using SPSS 19.0 statistical software (SPSS, Chicago, IL, USA).

\section{Results}

\section{Clinicopathological characteristics of CRC}

A total of 1490 cases of colorectal cancer were included. Of all investigated patients, there were $933(62.6 \%)$ males and 557 (37.4\%) females. The median age was 60 years (range 1691 years). Six $(0.4 \%)$ specimens were from metastatic sites, while $1484(99.6 \%)$ were from primary tumor sites through colectomy or colonoscopic biopsy. Regarding the location of the primary tumor, 1110 (74.5\%) tumors on the left side, 365 (24.5\%) tumors on the right side, and $15(1.0 \%)$ tumors on both sides of the colon. Histopathological examination identified $1435(96.3 \%)$ and $55(3.7 \%)$ cases with well to moderately, and poorly differentiated tumors, respectively. A total of 650 patients $(43.6 \%)$ were stage I and II, and 840 patients (56.4\%) were stage III and IV according to the 7th American Joint Committee on Cancer (AJCC)/International Union Against Cancer (UICC) staging system (Table 1).

We further analyzed clinicopathological characteristics of mCRC. For 244 patients with mCRC, 150 (61.5\%) were male, and $94(38.5 \%)$ were female; the median age of this cohort was 58 years (range 23-85 years). A total of 163 (66.8\%) patients presented with liver metastases, making it the most common metastatic site. A total of $146(59.8 \%)$ patients received an RAS gene test, and 58 (39.7\%) of them were found to harbor RAS mutations. A total of 228 (93.4\%) patients underwent DNA mismatch repair or microsatellite instability tests, and 10 (4.4\%) cases displayed DNA mismatch repair deficiency (MMR-D) or high-level microsatellite instability (MSI-H) phenotypes (Supplementary Table 1).

\section{HER2 assessment in CRC}

\section{The GEA criteria}

Of the 1490 CRC specimens assessed by IHC, 959 (64.4\%), $410(27.5 \%), 87(5.8 \%)$, and 34 (2.3\%) had HER2 scores of 0 , $1+, 2+$, and $3+$, respectively, according to the criteria for gastroesophageal adenocarcinoma (Fig. 1a-d). FISH was performed for the 87 cases with IHC score 2+, of which 78 cases had negative amplification of the HER 2 gene, and 9 cases had positive amplification of the HER2 gene (Fig. 1e, f). Overall, $1447(97.1 \%)$ cases were evaluated as HER2 negative, and 43 (2.9\%) cases were HER2 positive (Table 2). For the mCRC cohort, 167 (68.4\%), 56 (23.0\%), $12(4.9 \%)$, and $9(3.7 \%)$ cases assessed by IHC showed HER2 scores of $0,1+, 2+$, and $3+$, respectively. FISH examination for the 12 cases of HER 2 score $2+$ showed that only one case had positive amplification of the HER2 gene. In total, 234 (95.9\%) cases were evaluated as HER2 negative and only $10(4.1 \%)$ cases were HER2 positive (Supplementary Table 2).

\section{The HERACLES criteria}

Further, FISH was performed for 4 cases with a HER2 score $3+$ but in less than $50 \%$ of the tumor cells, as determined by IHC, according to the colorectal cancer-specific HERACLES diagnostic criteria [14], and the FISH results were all negative. We checked the 4 cases, two of them were with a 3+ HER2 score in $10 \%$ of the tumor cells by IHC, the intensity of the third one was moderate but also classified into $3+$ score after discussion and the percentage was $30 \%$ of the tumor cells, and the fourth one was with a $3+$ HER 2 score in $40 \%$ of the tumor cells by IHC. Owing to the heterogeneity of tumor and the definition of HER2 amplification by FISH according to the HERACLES criteria, the 4 cases were classified to HER2 negative. Among the 4 cases, there was one patient with stage I, two patients with stage II, and one patient with stage III disease. Hence, $2.6 \%$ (39/1490) of cases showed HER2 positivity in all CRCs according to the HERACLES criteria (Table 2). The positivity of HER2 for mCRC cohort was $4.1 \%(10 / 244)$, the same according to both criteria (Supplementary Table 2). 
Table 1 Relationship between the expression of HER2 and the clinicopathological characteristics of colorectal cancer according to the two criteria

\begin{tabular}{|c|c|c|c|c|c|c|c|}
\hline \multirow[t]{2}{*}{ Variables } & \multicolumn{4}{|c|}{ The criteria for gastroesophageal adenocarcinoma } & \multicolumn{3}{|c|}{ The HERACLES diagnostic criteria } \\
\hline & All patients & HER2 negative & HER2 positive & $P$ value & HER2 negative & HER2 positive & $P$ value \\
\hline Gender & & & & 0.149 & & & 0.179 \\
\hline Male & 933 & 911 & 22 & & 913 & 20 & \\
\hline Female & 557 & 536 & 21 & & 538 & 19 & \\
\hline Age (years) & & & & 0.867 & & & 0.860 \\
\hline$\leq 65$ & 1050 & 1019 & 31 & & 1023 & 27 & \\
\hline$>65$ & 440 & 428 & 12 & & 428 & 12 & \\
\hline \multicolumn{8}{|l|}{ Primary tumor location } \\
\hline Left-sided & 1110 & 1073 & 37 & $0.108^{\mathrm{a}}$ & 1075 & 35 & $0.037^{\mathrm{a}}$ \\
\hline Right-sided & 365 & 359 & 6 & & 361 & 4 & \\
\hline Both-sided & 15 & 15 & 0 & & 15 & 0 & \\
\hline Differentiation grade & & & & 1.0 & & & 1.0 \\
\hline Well-moderate & 1435 & 1393 & 42 & & 1397 & 38 & \\
\hline Poor & 55 & 54 & 1 & & 54 & 1 & \\
\hline \multicolumn{8}{|c|}{ Regional lymph node metastasis } \\
\hline No. of cases evaluated & $1429^{b}$ & & & 0.062 & & & 0.035 \\
\hline Absent & 681 & 667 & 14 & & 669 & 12 & \\
\hline Present & 748 & 719 & 29 & & 721 & 27 & \\
\hline TNM stage & & & & 0.086 & & & 0.022 \\
\hline I-II & 650 & 637 & 13 & & 640 & 10 & \\
\hline III-IV & 840 & 810 & 30 & & 811 & 29 & \\
\hline \multicolumn{8}{|l|}{ MSI test } \\
\hline No. of cases evaluated & $1452^{c}$ & & & 0.247 & & & 0.111 \\
\hline MSI-H (MMR-D) & 126 & 125 & 1 & & 126 & 0 & \\
\hline MSS/MSI-L (MMR-P) & 1326 & 1289 & 37 & & 1292 & 34 & \\
\hline \multicolumn{8}{|l|}{ RAS gene } \\
\hline No. of cases evaluated & $201^{\mathrm{d}}$ & & & 0.001 & & & 0.001 \\
\hline RAS wild type & 112 & 101 & 11 & & 101 & 11 & \\
\hline RAS mutation & 89 & 89 & 0 & & 89 & 0 & \\
\hline \multicolumn{8}{|l|}{ Ki-67 } \\
\hline No. of cases evaluated & $1423^{\mathrm{e}}$ & & & 0.507 & & & 0.463 \\
\hline$\leq 15 \%$ & 88 & 85 & 3 & & 85 & 3 & \\
\hline$>15 \%$ & 1335 & 1300 & 35 & & 1304 & 31 & \\
\hline
\end{tabular}

${ }^{\mathrm{a}} P$ was evaluated between left and right sided

${ }^{\mathrm{b}}$ Regional lymph node metastasis of 58 cases was difficult to identify due to the neoadjuvant chemotherapy or/and radiotherapy ( 3 cases) and at stage IV disease (58 cases)

${ }^{\mathrm{c}} 38$ cases did not receive MSI test

${ }^{\mathrm{d}} 201$ cases received RAS gene test

${ }^{\mathrm{e}} 67$ cases did not receive Ki-67 test

HER2, human epidermal growth factor receptor 2; MSI, microsatellite instability; $M S I-H$, high-level microsatellite instability; $M M R$ - $D$, mismatch repair deficiency; MSS, microsatellite stability; $M S I-L$, low-level microsatellite instability; $M M R-P$, mismatch repair proficiency

\section{Correlation of HER2 expression with clinicopathological characteristics in all CRCs}

\section{The GEA criteria}

The HER2 positivity rate was more common in female patients (37.7\% vs. $23.6 \%)$; however, the difference was not statistically significant $(P=0.149)$. In that HER2 positivity only presented in patients with RAS wild type, HER2 status was significantly correlation with RAS gene $(P=0.001)$. However, it was no correlation between HER2 status with clinicopathological variables such as primary tumor location $(P=0.108)$, tumor differentiation grade $(P=1.0)$, and TNM stage $(P=0.086)$ in all CRCs according to the GEA criteria (Table 1).

\section{The HERACLES criteria}

According to the HERACLES criteria, HER2 positivity was significantly correlation with primary tumor location $(P=$ 0.037), regional lymph node metastasis $(P=0.035)$, TNM stage $(P=0.022)$, and RAS status $(P=0.001)$ (Table 1$)$.

\section{Correlation of HER2 expression with clinicopathological characteristics in mCRCs}

Since the positivity was the same according to both criteria, the association of HER2 status and clinicopathological variables was further explored in mCRC. One hundred forty-six patients received an RAS gene test, and HER2 positivity was 

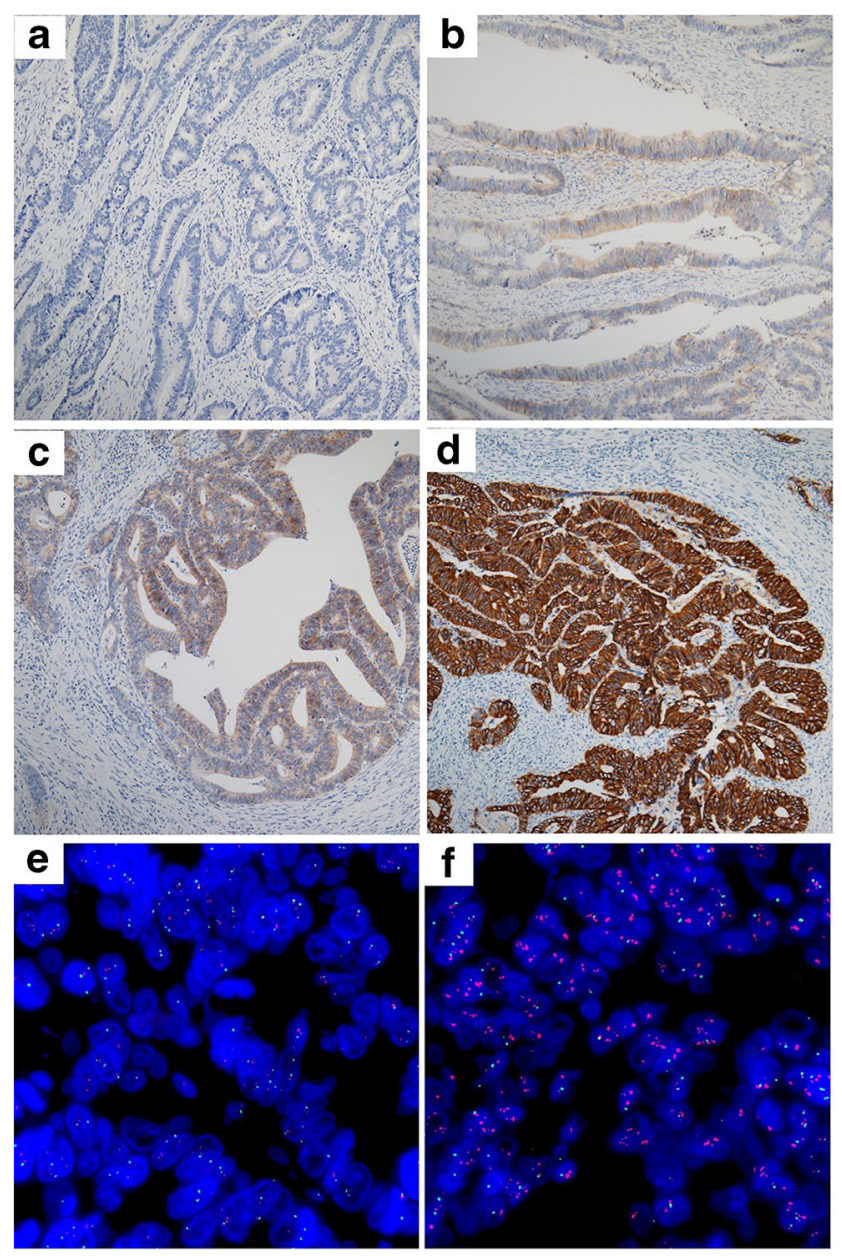

Fig. 1 HER2 evaluation by immunohistochemistry and fluorescence in situ hybridization in colorectal cancer specimens. Representative immunostaining intensity of tumor cells: $0 /$ none (a), $1+$ / faint (b), $2+$ / week to moderate (c), $3+/$ strong (d). Representative negative (e) and positive (f) amplification of HER2 gene statistically significant associated with RAS gene $(P=0.042)$. Yet the results were not significant for primary tumor site $(P=0.122)$ and metastatic site $(P=0.5)$ (Supplementary Table 1).

\section{Survival analysis}

The median follow-up time was 11.1 months (range 2.665.4 months). DFS was evaluated in patients with stage IIIII CRC who were treated with surgery. No significant difference was observed between the HER2-positive and HER2negative groups in terms of DFS either according to the GEA criteria $(P=0.052)$ (Fig. 2a). However, HER2 status was associated with DFS according to the HERACLES criteria $(P=0.048)$ (Fig. 2b), yet multivariate analysis showed that HER2 status was not an independent prognosis for DFS (Supplementary Table 3).

RFS was evaluated in patients with mCRC. A total of 127 patients presented with PD after first-line treatment during the follow-up period. The median PFS was 13.3 months for all mCRCs. The median PFS was 15.4 months for patients with HER2 negativity, while it was 23.7 months in patients with HER2 positivity. No significant difference was observed between the HER2-positive and HER2-negative groups in terms of PFS in mCRC $(P=0.919)$ (Fig. 2c).

\section{Discussion}

In this large Chinese cohort study, we evaluated HER2 status in CRC based on two different criteria and found that HER2 positivity in colorectal adenocarcinoma was quite low in Chinese population, with a rate of $2.9 \%$ according to the HER2 diagnostic criteria for gastroesophageal adenocarcinoma, and a rate of $2.6 \%$ according to the HERACLES criteria. HER2
Table 2 HER2 assessment in colorectal cancer according to different systems

\begin{tabular}{|c|c|c|c|c|c|}
\hline & & \multicolumn{2}{|c|}{$\begin{array}{l}\text { The criteria for gastroesophageal } \\
\text { adenocarcinoma }\end{array}$} & \multicolumn{2}{|c|}{ The HERACLES diagnostic criteria } \\
\hline \multirow[t]{5}{*}{ HER2 (IHC) } & Valid/missing & $1490 / 0$ & & 1490 & \\
\hline & 0 & 959 & $64.4 \%$ & 959 & $64.4 \%$ \\
\hline & $1+$ & 410 & $27.5 \%$ & 410 & $27.5 \%$ \\
\hline & $2+$ & 87 & $5.8 \%$ & 87 & $5.8 \%$ \\
\hline & $3+$ & 34 & $2.3 \%$ & 34 & $2.3 \%$ \\
\hline \multirow[t]{3}{*}{ HER2 (FISH) } & Valid/missing & $87 / 1403$ & & $91 / 1$ & \\
\hline & Not amplified & 78 & $89.7 \%$ & 82 & $90.1 \%$ \\
\hline & Amplified & 9 & $10.3 \%$ & 9 & $9.9 \%$ \\
\hline \multirow[t]{3}{*}{ HER2 status } & Valid/missing & $1490 / 0$ & & 1490 & \\
\hline & Negative & 1447 & $97.1 \%$ & 1451 & $97.4 \%$ \\
\hline & Positive & 43 & $2.9 \%$ & 39 & $2.6 \%$ \\
\hline
\end{tabular}

HER2, human epidermal growth factor receptor 2; IHC, immunohistochemistry; FISH, fluorescent in situ hybridization 


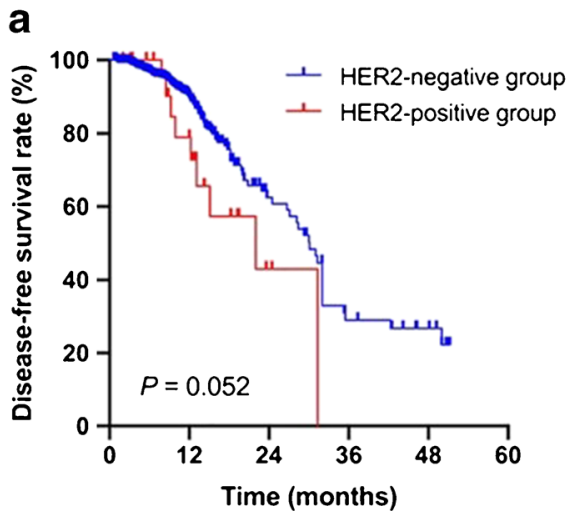

Fig. 2 Kaplan-Meier curves of disease-free survival in HER2-positive and HER2-negative patients with stage II-III disease treated with surgery according to the HER2 diagnostic criteria for gastroesophageal
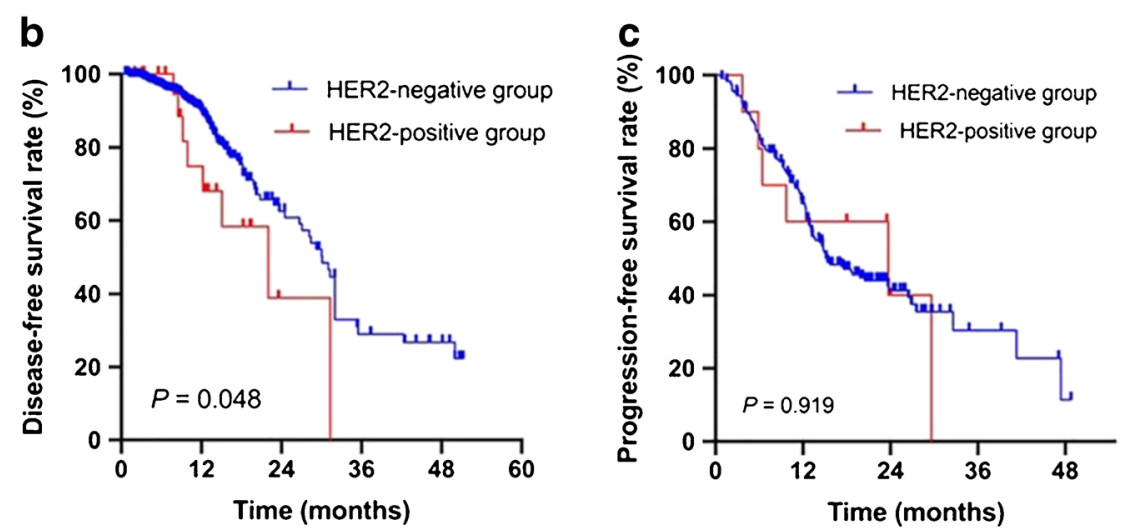

adenocarcinoma (a) and the HERACLES diagnostic criteria (b). Kaplan-Meier curves of progression-free survival in HER2-positive and HER2-negative patients with metastatic colorectal cancer (c) status was associated with clinical variables and DFS in patients with stage II-III CRC according to the HERACLES criteria, yet, the results were not found according to the HER2 diagnostic criteria for gastroesophageal adenocarcinoma. However, HER2 status had no influence on RFS in patients with mCRC.

Various research groups have investigated the frequency of HER2 expression in CRC, with a variability ranging from 1.3 to $82 \%$ owing to different scoring systems [15-17]. Richman SD et al. [15] analyzed HER2 amplification/overexpression in 3256 patients from three colorectal cancer trials in the UK and found that the overexpression of HER2 was observed in 25 of 1914 (1.3\%) stage II-III tumors and 29 of 1342 (2.2\%) stage IV tumors. This small observed proportion of HER2 expression is in line with our obtained results for CRC. Since the standard of HER2 evaluation criteria was not established in CRC, the scoring system of HER2 in CRC of these studies usually used the criteria in gastroesophageal adenocarcinoma (GEA) [15-17, 23].

Valtorta E et al. [24] developed the HERACLES diagnostic criteria for HER2 in CRC: tumors with a 3+ HER2 score in more than $50 \%$ of cells by IHC or tumors with a $2+$ HER 2 score and a HER2:CEP17 ratio higher than two in more than $50 \%$ of cells by FISH. The significant difference from the criteria in GEA lies in the cutoff value for the IHC evaluation. Our study was the first to evaluate the two different scoring systems of HER2 for CRC in one large cohort. Further, we found HER2 positivity was associated with tumor location $(P=0.037)$, regional lymph node metastasis $(P=0.035)$, and tumor stage $(P=0.022)$ according to the HERACLES criteria, which were not present according to the GEA criteria. RAS gene test was performed in 201 patients of entire cohort, and HER2 positivity only presented in patients with RAS wild type $(P=0.001)$ based on both criteria. The result was reasonable due to HER2 and RAS protein are the upstream and down steam of MAP kinase pathway; therefore, HER2 positivity and RAS mutation were mutually exclusive. Moreover, we found HER2 positivity was mainly presented in patients with mismatch repair proficiency (MMR-P), which also can be explained by sporadic MSI. CRC is associated in $60 \%$ of tumors with BRAF mutation and BRAF is the downstream of HER2 protein [7]. Previous studies have reported that the HER2 expression was significantly correlated with tumor size, histological differentiation, lymph node metastases, and tumor stage [25-28]. In contrast to these studies, other researchers have found no such association [29, 30]. These studies analyzed the association using the GEA criteria to identify HER2 positivity, and the results were equivocal. However, our results found that based on the HERACLES criteria, HER2 positivity was more common in CRC patients with left-sided, presence of regional lymph node metastasis, advanced stage, and RAS wild type.

To further explore the impact of HER2 on survival based on different scoring systems, we selected patients with stage II-III CRC who receive surgery as initial treatment; the results showed that gender, vascular invasion, perineural invasion, HER2 status, and tumor stage were associated with DFS, yet multivariate analysis showed only tumor stage was the independent prognostic factor according to the HERACLES criteria. No such association between HER2 status and DFS was found based on the GEA criteria, but the $P$ value was 0.052 . There were only 3 cases in which HER2 status was positive in the GEA criteria and negative in the HERACLES criteria in patients with stage II-III disease. Among them, one patient presented lung recurrence at 13.1 months after surgery, and the rest two did not present recurrence at the time of censor. Therefore, it was reasonable that the results were marginal and HER2 positivity was a factor associated with worse DFS according to the HERACLES criteria. The negative prognostic impact of HER2 overexpression was also be discovered in other studies [19, 27]. The different results between HER2 status and clinicopathological factors, survival in CRC indicated that the HERACLES criteria would be a favorable scoring system for HER2 assessment of CRC.

Considering that anti-HER2 treatment was applied in mCRC, we further evaluated the correlation between HER2 expression and clinicopathological factors in $\mathrm{mCRC}$, and influence on PFS. It is interesting that HER2 status turned out to be the same according to the two criteria. Moreover, all eight cases 
which present $3+$ IHC score were presented in more than $50 \%$ of the cells. The results indicated that overexpression of HER2 protein was low in mCRC but it was highly expressed in more than half the tumor cells in our study. Still, HER2 status was found all negative in RAS mutant group. The prognostic role of HER2 in mCRC remains uncertain. Ingold Heppner B et al. [27] considered that although statistically not significant $(P=$ 0.208), HER2-positive colorectal carcinomas displayed a tendency to poorer overall survival. We found that HER2 expression had no impact on the PFS of mCRC patients. Although our study did not find a difference in PFS between the HER2positive and HER2-negative groups, the mean PFS was longer in the HER2-positive group than in the HER2-negative group (23.7 months vs. 15.4 months). It seemed that HER2 positivity played a positive impact on survival of patients with mCRC; however, a negative impact on patients with stage II-III CRC. The exact role of HER2 on the process of CRC molecular pathogenesis need to be further explored.

There were several limitations of this study. First, the data were from a single center, and the results only reflected HER2 expression in southern China due to patients in our hospital mainly came from provinces of south China. Second, we did not perform FISH on cases with an IHC score of $3+$ in more than $50 \%$ of the tumor cells for both criteria and considered these cases as HER2 positive. Therefore, it was difficult to evaluate the accordance rate of IHC and FISH in cases with IHC score $3+$, as well as positive agreement and negative agreement rates. Third, as a result of the limited follow-up time, we did not evaluate overall survival in the entire cohort. Finally, this was a descriptive, retrospective study, and we did not investigate the effects of anti-HER2 treatment in mCRC.

Our study indicated that the frequency of HER2 overexpression or amplification was low in CRC in Chinese population. HER2 status evaluated by the HERACLES criteria showed clinicopathological association and survival impact on CRC, not by the GEA criteria. Additionally, HER2 positivity in mCRC was identical according to both criteria. Our findings provided a rationale for further evaluation of HER2 in CRC based on the HERACLES criteria and the HER2 diagnostic criteria for gastroesophageal adenocarcinoma.

Acknowledgments We thank the central laboratory of Department of Pathology at Sun Yat-sen University Cancer Center for supporting the part of HER2 IHC test in this study.

\footnotetext{
Authors' contributions Furong Liu and Chao Ren performed data acquisition, statistical analyses, and wrote the first draft of the paper. Ying Jing and Zixian Wang performed data acquisition and contributed to the paper writing. Shaoyan Xi, Caiyu He, and Fang Wang performed the immunohistochemical and fluorescence in situ hybridization analysis. Rui-hua Xu reviewed the manuscript. Feng Wang conceived and designed the study, and took full responsibility for the work as a whole, including the study design, access to data, and the decision to submit and publish the manuscript. All authors gave final approval for publication.
}

Funding information This study was funded by, in part, by the National Key Research and Development Program of China (2017YFC1308900), National Natural Science Foundation of China (81872011), and the Sun Yat-sen University Clinical Research 5010 Program (2018014), Guangzhou Health and Medical Collaborative Innovation Project (201704020220). Feng Wang is the Young Physician Scientist Program of Sun Yat-sen University Cancer Center.

\section{Compliance with ethical standards}

Conflict of interest The authors have declare that they have no competing interests.

Informed consent The Institutional Review Board approved this study.

Open Access This article is distributed under the terms of the Creative Commons Attribution 4.0 International License (http:// creativecommons.org/licenses/by/4.0/), which permits unrestricted use, distribution, and reproduction in any medium, provided you give appropriate credit to the original author(s) and the source, provide a link to the Creative Commons license, and indicate if changes were made.

\section{References}

1. Bray F, Ferlay J, Soerjomataram I, Siegel RL, Torre LA, Jemal A (2018) Global cancer statistics 2018: GLOBOCAN estimates of incidence and mortality worldwide for 36 cancers in 185 countries. CA Cancer J Clin 68(6):394 424. https://doi.org/10.3322/caac.21492

2. Chen W, Sun K, Zheng R, Zeng H, Zhang S, Xia C, Yang Z, Li H, Zou X, He J (2018) Cancer incidence and mortality in China, 2014. Chin J Cancer Res 30(1):1-12. https://doi.org/10.21147/j.issn. 1000-9604.2018.01.01

3. Leufkens AM, van den Bosch MA, van Leeuwen MS, Siersema PD (2011) Diagnostic accuracy of computed tomography for colon cancer staging: a systematic review. Scand J Gastroenterol 46(78):887-894. https://doi.org/10.3109/00365521.2011.574732

4. Brenner H, Kloor M, Pox CP (2014) Colorectal cancer. Lancet 383(9927):1490-1502. https://doi.org/10.1016/S0140-6736(13) 61649-9

5. Arnold D, Seufferlein T (2010) Targeted treatments in colorectal cancer: state of the art and future perspectives. Gut 59(6):838-858. https://doi.org/10.1136/gut.2009.196006

6. Karapetis CS, Khambata-Ford S, Jonker DJ, O'Callaghan CJ, Tu D, Tebbutt NC, Simes RJ, Chalchal H, Shapiro JD, Robitaille S, Price TJ, Shepherd L, Au HJ, Langer C, Moore MJ, Zalcberg JR (2008) $\mathrm{K}$-ras mutations and benefit from cetuximab in advanced colorectal cancer. N Engl J Med 359(17):1757-1765. https://doi.org/10.1056/ NEJMoa0804385

7. Sanz-Garcia E, Argiles G, Elez E, Tabernero J (2017) BRAF mutant colorectal cancer: prognosis, treatment, and new perspectives. Ann Oncol 28(11):2648-2657. https://doi.org/10.1093/annonc/mdx401

8. Mendelsohn J, Baselga J (2006) Epidermal growth factor receptor targeting in cancer. Semin Oncol 33(4):369-385. https://doi.org/10. 1053/j.seminoncol.2006.04.003

9. Slamon DJ, Clark GM, Wong SG, Levin WJ, Ullrich A, McGuire WL (1987) Human breast cancer: correlation of relapse and survival with amplification of the HER-2/neu oncogene. Science 235(4785):177-182. https://doi.org/10.1126/science.3798106

10. Barros-Silva JD, Leitao D, Afonso L, Vieira J, Dinis-Ribeiro M, Fragoso M, Bento MJ, Santos L, Ferreira P, Rego S, Brandao C, Carneiro F, Lopes C, Schmitt F, Teixeira MR (2009) Association of 
ERBB2 gene status with histopathological parameters and diseasespecific survival in gastric carcinoma patients. Br J Cancer 100(3): 487-493. https://doi.org/10.1038/sj.bjc.6604885

11. Terashima M, Kitada K, Ochiai A, Ichikawa W, Kurahashi I, Sakuramoto S, Katai H, Sano T, Imamura H, Sasako M, Group A-G (2012) Impact of expression of human epidermal growth factor receptors EGFR and ERBB2 on survival in stage II/III gastric cancer. Clin Cancer Res 18(21):5992-6000. https://doi.org/10. 1158/1078-0432.CCR-12-1318

12. Mendes D, Alves C, Afonso N, Cardoso F, Passos-Coelho JL, Costa L, Andrade S, Batel-Marques F (2015) The benefit of HER2-targeted therapies on overall survival of patients with metastatic HER2-positive breast cancer-a systematic review. Breast Cancer Res 17:140. https://doi.org/10.1186/s13058-015-0648-2

13. Bang YJ, Van Cutsem E, Feyereislova A, Chung HC, Shen L, Sawaki A, Lordick F, Ohtsu A, Omuro Y, Satoh T, Aprile G, Kulikov E, Hill J, Lehle M, Ruschoff J, Kang YK, To GATI (2010) Trastuzumab in combination with chemotherapy versus chemotherapy alone for treatment of HER2-positive advanced gastric or gastro-oesophageal junction cancer (ToGA): a phase 3, openlabel, randomised controlled trial. Lancet 376(9742):687-697. https://doi.org/10.1016/S0140-6736(10)61121-X

14. Sartore-Bianchi A, Trusolino L, Martino C, Bencardino K, Lonardi S, Bergamo F, Zagonel V, Leone F, Depetris I, Martinelli E, Troiani T, Ciardiello F, Racca P, Bertotti A, Siravegna G, Torri V, Amatu A, Ghezzi S, Marrapese G, Palmeri L, Valtorta E, Cassingena A, Lauricella C, Vanzulli A, Regge D, Veronese S, Comoglio PM, Bardelli A, Marsoni S, Siena S (2016) Dual-targeted therapy with trastuzumab and lapatinib in treatment-refractory, KRAS codon 12/ 13 wild-type, HER2-positive metastatic colorectal cancer (HERACLES): a proof-of-concept, multicentre, open-label, phase 2 trial. Lancet Oncol 17(6):738-746. https://doi.org/10.1016/ S1470-2045(16)00150-9

15. Richman SD, Southward K, Chambers P, Cross D, Barrett J, Hemmings G, Taylor M, Wood H, Hutchins G, Foster JM, Oumie A, Spink KG, Brown SR, Jones M, Kerr D, Handley K, Gray R, Seymour M, Quirke P (2016) HER2 overexpression and amplification as a potential therapeutic target in colorectal cancer: analysis of 3256 patients enrolled in the QUASAR, FOCUS and PICCOLO colorectal cancer trials. J Pathol 238(4):562-570. https://doi.org/10. 1002/path.4679

16. Blok EJ, Kuppen PJ, van Leeuwen JE, Sier CF (2013) Cytoplasmic overexpression of HER2: a key factor in colorectal cancer. Clin Med Insights Oncol 7:41-51. https://doi.org/10.4137/CMO.S10811

17. McKay JA, Loane JF, Ross VG, Ameyaw MM, Murray GI, Cassidy J, McLeod HL (2002) c-erbB-2 is not a major factor in the development of colorectal cancer. Br J Cancer 86(4):568-573. https://doi.org/10.1038/sj.bjc.6600127

18. Park JS, Yoon G, Kim HJ, Park SY, Choi GS, Kang MK, Kim JG, Jang JS, Seo AN (2018) HER2 status in patients with residual rectal cancer after preoperative chemoradiotherapy: the relationship with molecular results and clinicopathologic features. Virchows Arch 473(4):413-423. https://doi.org/10.1007/s00428-018-2409-y

19. Kapitanovic S, Radosevic S, Kapitanovic M, Andelinovic S, Ferencic Z, Tavassoli M, Primorac D, Sonicki Z, Spaventi S, Pavelic K, Spaventi R (1997) The expression of p185(HER-2/ neu) correlates with the stage of disease and survival in colorectal cancer. Gastroenterology 112(4):1103-1113. https://doi.org/10. 1016/s0016-5085(97)70120-3

20. Conradi LC, Styczen H, Sprenger T, Wolff HA, Rodel C, Nietert M, Homayounfar K, Gaedcke J, Kitz J, Talaulicar R, Becker H, Ghadimi M, Middel P, Beissbarth T, Ruschoff J, Liersch T (2013) Frequency of HER-2 positivity in rectal cancer and prognosis. Am J
Surg Pathol 37(4):522-531. https://doi.org/10.1097/PAS. 0b013e318272ff4d

21. Bartley AN, Washington MK, Colasacco C, Ventura CB, Ismaila N, Benson AB 3rd, Carrato A, Gulley ML, Jain D, Kakar S, Mackay HJ, Streutker C, Tang L, Troxell M, Ajani JA (2017) HER2 testing and clinical decision making in gastroesophageal adenocarcinoma: guideline from the College of American Pathologists, American Society for Clinical Pathology, and the American Society of Clinical Oncology. J Clin Oncol 35(4):446-464. https://doi.org/ 10.1200/JCO.2016.69.4836

22. Shi Q, de Gramont A, Grothey A, Zalcberg J, Chibaudel B, Schmoll HJ, Seymour MT, Adams R, Saltz L, Goldberg RM, Punt CJ, Douillard JY, Hoff PM, Hecht JR, Hurwitz H, Diaz-Rubio E, Porschen R, Tebbutt NC, Fuchs C, Souglakos J, Falcone A, Tournigand C, Kabbinavar FF, Heinemann V, Van Cutsem E, Bokemeyer C, Buyse M, Sargent DJ (2015) Individual patient data analysis of progression-free survival versus overall survival as a first-line end point for metastatic colorectal cancer in modern randomized trials: findings from the analysis and research in cancers of the digestive system database. J Clin Oncol 33(1):22-28. https:// doi.org/10.1200/JCO.2014.56.5887

23. Lee WS, Park YH, Lee JN, Baek JH, Lee TH, Ha SY (2014) Comparison of HER2 expression between primary colorectal cancer and their corresponding metastases. Cancer Med 3(3):674-680. https://doi.org/10.1002/cam4.228

24. Valtorta E, Martino C, Sartore-Bianchi A, Penaullt-Llorca F, Viale G, Risio M, Rugge M, Grigioni W, Bencardino K, Lonardi S, Zagonel V, Leone F, Noe J, Ciardiello F, Pinto C, Labianca R, Mosconi S, Graiff C, Aprile G, Frau B, Garufi C, Loupakis F, Racca P, Tonini G, Lauricella C, Veronese S, Truini M, Siena S, Marsoni S, Gambacorta M (2015) Assessment of a HER2 scoring system for colorectal cancer: results from a validation study. Mod Pathol 28(11):1481-1491. https://doi.org/10.1038/modpathol. 2015.98

25. Li Q, Wang D, Li J, Chen P (2011) Clinicopathological and prognostic significance of HER-2/neu and VEGF expression in colon carcinomas. BMC Cancer 11:277. https://doi.org/10.1186/14712407-11-277

26. Shabbir A, Mirza T, Khalid AB, Qureshi MA, Asim SA (2016) Frequency of Her2/neu expression in colorectal adenocarcinoma: a study from developing South Asian country. BMC Cancer 16(1): 855. https://doi.org/10.1186/s12885-016-2912-y

27. Ingold Heppner B, Behrens HM, Balschun K, Haag J, Kruger S, Becker T, Rocken C (2014) HER2/neu testing in primary colorectal carcinoma. Br J Cancer 111(10):1977-1984. https://doi.org/10. 1038/bjc.2014.483

28. Sun SJ, Lin Q, Sun Q, Li J, Zhang XY, Tan ZG, Song Y, Guo YT, Li Y (2016) High HER-2 protein levels correlate with clinicopathological features in colorectal cancer. J Cancer Res Ther 12(1):323333. https://doi.org/10.4103/0973-1482.155977

29. Torabizadeh Z, Nosrati A, Tahvildari S (2016) Human epidermal growth factor receptor expression in colorectal cancer and its relationship with clinicopathological characteristics. Middle East J Dig Dis 8(1):24-30. https://doi.org/10.15171/mejdd.2016.03

30. Karaca H, Deniz K, Berk V, Inanc M, Ozkan M (2012) Association of human epidermal growth factor receptor-2 expression and clinicopathological findings in patients with colorectal cancer. Asian Pac J Cancer Prev 13(12):6221-6225. https://doi.org/10.7314/ apjcp.2012.13.12.6221

Publisher's note Springer Nature remains neutral with regard to jurisdictional claims in published maps and institutional affiliations. 